\title{
THE PERIODICITY TO A KIND OF GENERALIZED COLLATZ PROBLEM
}

\section{SENSEN CHEN, QING-YOU SUN and YUSHU ZHU}

Hangzhou Normal University

Hangzhou 311121

P. R. China

e-mail: qysun@hznu.edu.cn

\begin{abstract}
The Collatz problem is related to the fixed point problem, and is widely used in mathematics. It has attracted a wide range of mathematics enthusiasts, but is still difficult to solve. So, this article aimed to study the extension of the Collatz problem, more widely, in $k$-adic. We define a new sequence called $\mathcal{Z}$ transformation sequence. Under a suitable assumptions, we can prove that the limit set of the $\mathcal{Z}$ transformation sequence must be $M=\{1,2\}$.
\end{abstract}

\section{Introduction}

The Collatz problem has been widely studied in the past 100 years, and many achievements with great value have been obtained, although the Collatz problem cannot be effectively solved. However, this does not affect the important position of the Collatz problem in mathematics.

2010 Mathematics Subject Classification: 11A99, 11A67, $11 \mathrm{~B} 83$.

Keywords and phrases: Collatz problem, $k$-adic, $\mathcal{Z}$ transformation, limit set.

Received August 21, 2019

(ㄷ) 2019 Scientific Advances Publishers 
The essence of the Collatz problem is a fixed-point problem, and its research results are important for solving fixed-point problems. Fixedpoint problems are an important foundation for modern analysis and topology, so the study of the Collatz problem is of great value. Moreover, it is widely used and has important connection with dynamic system, fractal geometry and other fields. It is also the theoretical basis of cryptography research.

The Collatz problem was proposed by Collatz [1], a German mathematician, in 1937. He conjectured the following number-theoretic function:

$$
f(x)= \begin{cases}\frac{x}{2}, & \text { when } x \text { is even } \\ \frac{3 x+1}{2}, & \text { when } x \text { is odd }\end{cases}
$$

There exists a finite positive integer $k$, s.t., $f^{n}(x) \in\{1,4,2\}$ when $n \geq k$, and $x \in \mathbb{N}$. We call this problem Collatz conjecture. This problem mainly studies the periodicity of sequence transformation.

The Collatz problem can be stated in the form of 2-adic [2]. The integer in $\mathbb{Z}_{2}$ can be expressed as

$$
\alpha=a_{m-1} 2^{m-1}+a_{m-2} 2^{m-2}+\cdots+a_{1} \cdot 2+a_{0},
$$

where $a_{i}=0$, or $1,0 \leq i<m-1,1 \leq m<\infty$. It called the 2 -adic form of $\alpha$.

One can define congruence on $\mathbb{Z}_{2}$ by $\alpha \equiv \beta\left(\bmod 2^{m}\right)$ if the first $m$ 2 -adic digits of $\alpha$ and $\beta$ agree. Addition and multiplication on $\mathbb{Z}_{2}$ are given by

$$
\begin{gathered}
(X)_{2}=(\alpha+\beta)_{2}=X\left(\bmod 2^{k}\right)=\alpha\left(\bmod 2^{k}\right)+\beta\left(\bmod 2^{k}\right), \\
(X)_{2}=(\alpha \beta)_{2}=X\left(\bmod 2^{k}\right)=\alpha\left(\bmod 2^{k}\right) \cdot \beta\left(\bmod 2^{k}\right) .
\end{gathered}
$$


Now, one can extend the definition of the function $T: \mathbb{Z} \rightarrow \mathbb{Z}$ given by (1) to $T: \mathbb{Z}_{2} \rightarrow \mathbb{Z}_{2}$ by

$$
T(x)= \begin{cases}\frac{x}{2}, & x \equiv 0(\bmod 2), \\ \frac{3 x+1}{2}, & x \equiv 1(\bmod 2) .\end{cases}
$$

Heppner [3] got an important corollary and extended it to the form of module $p$. In [3], the following mapping is given

$$
T(n)=T_{m, p, j}(n) \begin{cases}\frac{m n+r_{j}}{p}, & n \equiv j(\bmod p), \\ \frac{n}{p}, & n \equiv 0(\bmod p),\end{cases}
$$

where $(m, p)=1$ with $p>2$, and $r_{j} \equiv-m_{j}(\bmod p)$. Meanwhile, the transform are shown to depend on relative sizes of $m$ and $p$.

Matthews [4] studied some maps on the rings of integers in an algebraic number field, such as

$$
U(\alpha)= \begin{cases}\frac{(1-\sqrt{2}) \alpha}{\sqrt{2}}, & \alpha \equiv 0(\bmod 2), \\ \frac{3 \alpha+1}{\sqrt{2}}, & \alpha \equiv 1(\bmod 2) .\end{cases}
$$

Certainly, there are many other research results of such problems and their extended problems ([5]-[8]), especially through computers to solve such problems. This article mainly explores a more extensive periodic problem of a particular sequence transformation based on the Collatz problem.

In the present article, we will extend the Collatz problem in $p$-adic. Then, we give a new transformation, called $\mathcal{Z}$ transformation. Through the study of the property of the $\mathcal{Z}$ transformation, we find that under some suitable assumptions, the $\mathcal{Z}$ transformation has a periodic characteristic. 


\section{Definition and Main Result}

For any given $m$-digit natural number $n$, it can be expressed in $k$-adic as

$$
n=a_{m-1} k^{m-1}+a_{m-2} k^{m-2}+\cdots+a_{1} k+a_{0},
$$

where $a_{i} \in \mathbb{Z}_{0}^{+}, 0 \leq a_{i}<k(0 \leq i \leq m-1)$, and $a_{m-1} \neq 0$.

We introduce the function

$$
f(x)=\left\{\begin{array}{cc}
\frac{(x+p-1)(x+2 p-1)}{p \cdot p}, & x \equiv 1(\bmod p), \\
\frac{x+p-2}{p}, & x \equiv 2(\bmod p), \\
\vdots & x=p-1(\bmod p), \\
\frac{x+1}{p}, & x \equiv 0(\bmod p) . \\
\frac{x}{p}, &
\end{array}\right.
$$

Definition 1. Let $\mathcal{Z}_{k}(n)=\sum_{i=0}^{m-1} f\left(a_{i}\right)$, where $f(x)$ is defined as (4).

Then, $\mathcal{Z}_{k}(n)$ is called the $\mathcal{Z}$ transformation of $n$ in $k$-adic

Definition 2. Denote $n_{j}=\mathcal{Z}_{k}^{j}(n),\left(j \in \mathbb{Z}_{0}^{+}\right)$. It is clear that, $\left\{n_{j}\right\}$ is a sequence, it is called $\mathcal{Z}$ transformation sequence in $k$-adic.

We give our main result as the following theorem.

Theorem 1. Under the assumptions

$$
p+2 \leq k<p^{2}-3 p+2, \quad \text { and } \quad p>5,
$$

the $\mathcal{Z}$ transformation sequence in $k$-adic has a limit set $M=\{1,2\}$, i.e., there exists a finite positive integer $\lambda$, subject to $n_{\mu} \in\{1,2\}$ when $\mu \geq \lambda$. 


\section{The Proof of Theorem 1}

To prove the Theorem 1, we give two lemmas first.

Lemma 1. The result of adding two k-adic numbers is the same as the result of adding them in decimal to become $k$-adic.

This conclusion is obvious (see [2]). We omit it.

Lemma 2. For any given m-digit natural number $n$, which is expressed in $k$-adic as (3), under the assumptions (5), $\mathcal{Z}_{k}(n)$ is a integer not exceeding $(m-1)$-digit in $k$-adic when $m \geq 3$.

Proof. Denote

$$
k=r p+s+1
$$

where $r, s \in \mathbb{N}$ and $1 \leq s \leq p$.

Then, by calculating the assumptions (5) are equivalent to

$$
1 \leq r \leq p-4,
$$

or

$$
(r+1)(r+2) \leq r p+1
$$

Due to $0 \leq a_{i} \leq k-1$, by the definition of $f(x)$ in (4), it obtains

$$
f\left(a_{i}\right) \leq f(r p+1)=(r+1)(r+2) .
$$

Thus, $\mathcal{Z}_{k}(n) \leq m(r+1)(r+2)$.

For $m=3$, by (5), (6), and (8), we have

$$
\mathcal{Z}_{k}(n) \leq 3(r+1)(r+2)<3(r p+1)=3(k-s)<3 k<k^{3-1} .
$$

For $m=i$, we assume that it holds

$$
\mathcal{Z}_{k}(n) \leq i(r+1)(r+2)<k^{i-1}
$$


Then, for $m=i+1$, it's obviously that

$$
\mathcal{Z}_{k}(n) \leq(i+1)(r+1)(r+2)<k^{i-1} \cdot \frac{i+1}{i}<k^{(i-1)+1} .
$$

Therefore, by mathematical induction, we have

$$
\mathcal{Z}_{k}(n)<k^{m-1}
$$

where $m \geq 3$. That means, $\mathcal{Z}_{k}(n)$ is an integer not exceeding $(m-1)$-digit in $k$-adic when $m \geq 3$. Thus, the proof of Lemma 2 is done.

Proof of Theorem 1. For any given $m$-digit natural number $n$, which is expressed in $k$-adic as (3), we will prove the theorem in three cases.

Case 1. $n$ is a single-digit number.

In this case, it is easy to show that $1 \leq n<k$, and $\mathcal{Z}_{k}(n)=f(n)$.

If $n \neq 1(\bmod p)$, by the definition of $f$, that is (4), we can easily get $\mathcal{Z}_{k}(n)=f(n)<n$. It implies that the $\mathcal{Z}$ transformation make $\mathcal{Z}_{k}(n)$ smaller than $n$, and $\mathcal{Z}_{k}(n)$ is still a single-digit number. Therefore, there exists a nonnegative integer $\lambda$, which satisfies $\mathcal{Z}_{k}^{\lambda}(n)=1$, or $\mathcal{Z}_{k}^{\lambda}(n) \equiv 1(\bmod p)$. The second case is just what we will discuss next.

If $n \equiv 1(\bmod p)$ and $n \neq 1$, let $n=\tilde{r} p+1$. So, by using $n<k$ and (6), we have $1 \leq \tilde{r} \leq r$.

Noting the definition of $f$, it can obtain that

$$
\mathcal{Z}_{k}(n)=f(n)=f(\tilde{r} p+1)=(\tilde{r}+1)(\tilde{r}+2) .
$$

Then, under the equivalent assumption (7) and (8),

$$
\mathcal{Z}_{k}(n)<\tilde{r} p+1=n,
$$


here, $1 \leq \tilde{r} \leq r \leq p-4$. Also, the $\mathcal{Z}$ transformation make $\mathcal{Z}_{k}(n)$ smaller than $n$, and $\mathcal{Z}_{k}(n)$ is still a single-digit number. It means, there exists a nonnegative integer $\lambda$, which satisfies $\mathcal{Z}_{k}^{\lambda}(n)=1$, or $\mathcal{Z}_{k}^{\lambda}(n) \neq 1$ $(\bmod p)$. The second case is exactly what we discussed earlier.

Hence, there exists a nonnegative integer $\lambda$, which satisfies $\mathcal{Z}_{k}^{\lambda}(n)=1$, when $n$ is a single-digit number.

Case 2. $n$ is a 2-digit number.

In this case, we denote $n=c k+d$, where $c \in \mathbb{N}, d \in \mathbb{Z}_{0}^{+}$, and $c, d<k$. Thus, $\mathcal{Z}_{k}(n)=f(c)+f(d)$.

Noting $f(1)=2$ and the conclusion in Case 1, under the assumption (5), it's easy to obtain that

$$
\mathcal{Z}_{k}(n)=f(c)+f(d) \leq(c+1)+(d+1)<c k+d=n .
$$

Therefore, there exists a nonnegative integer $\lambda$, which satisfies $\mathcal{Z}_{k}^{\lambda}(n)$ is a single-digit number. It will become Case 1.

Case 3. $n$ is an $m$-digit number, where $m \geq 3$.

In this case, by Lemma 2, we can easily get that there exists a nonnegative integer $\lambda, \lambda \leq m-2$, which satisfies $\mathcal{Z}_{k}^{\lambda}(n)$ is a 2 -digit number. It will become Case 2, then, Case 1.

Overall, it is not difficult to see that there will exist a nonnegative integer $\lambda$, which satisfies $\mathcal{Z}_{k}^{\lambda}(n)=1$. And noting $\mathcal{Z}_{k}(1)=f(1)=2$, $\mathcal{Z}_{k}(2)=f(2)=1$, therefore, for any positive integer $\mu$, which satisfies $\mu \geq \lambda$, we have $\mathcal{Z}_{k}^{\mu}(n) \in\{1,2\}$.

Hence, Theorem 1 is proved. 
Consider a positive integer in decimalism as

$$
\alpha=\overline{a_{m-1} a_{m-2} \ldots a_{1} a_{0}},
$$

where $a_{i} \in \mathbb{Z}_{0}^{+}, 0 \leq a_{i} \leq 9(0 \leq i \leq m-1)$, and $a_{m-1} \neq 0$. As a direct result of Theorem 1 , we can easily obtain the following corollary.

Corollary 1. For any positive integer $\alpha$ in decimalism, that is the expression (10), the $\mathcal{Z}$ transformation sequence $\left\{\mathcal{Z}_{10}^{j}(\alpha), j \geq 0\right\}$ has a limit set $M=\{1,2\}$ when $p \in\{6,7,8\}$ in (4).

\section{Examples}

In this section, we give some examples for different $n, k$, and $p$, to see the periodic characteristic of the $\mathcal{Z}$ transformation.

Example 1. Take $n=9815671, k=16$, and $p=8$, this set satisfies the assumptions (5).

Example 2. Take $n=71517, k=10$, and $p=6$, this set satisfies the requirement of Theorem 1, also Corollary 1.

We can see from Figure 1 that by finite $\mathcal{Z}$ transformation, $n=987654321$ and $n=987654321$ will become 1 in their respective cases. 
THE PERIODICITY TO A KIND OF ...

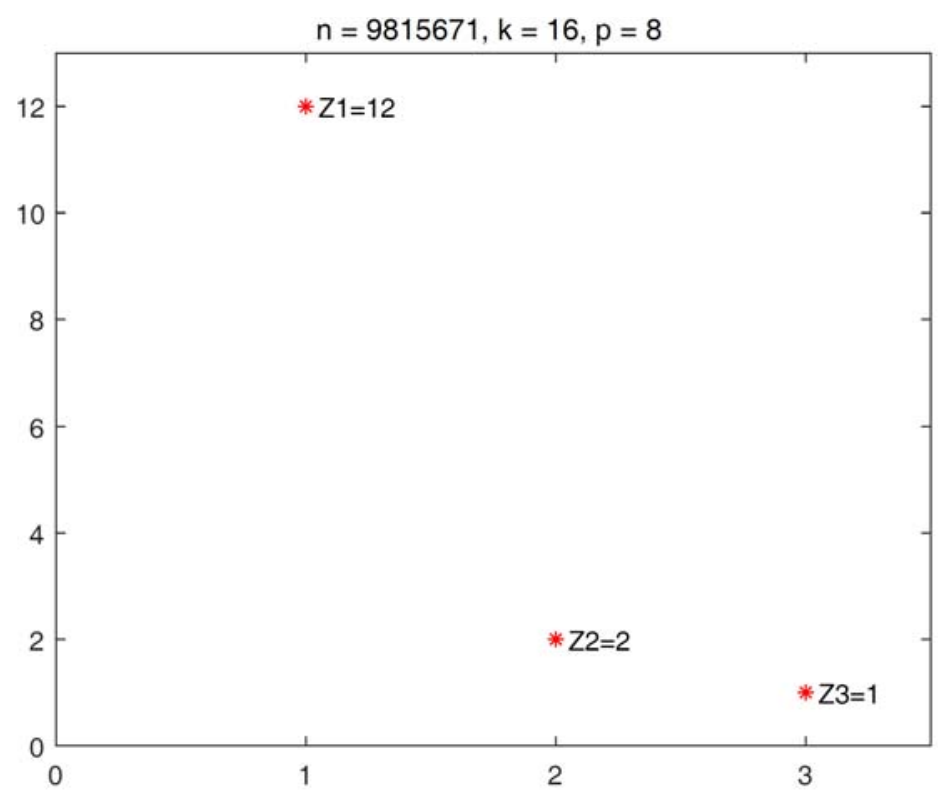

(a) $n=9815671, k=16, p=8$

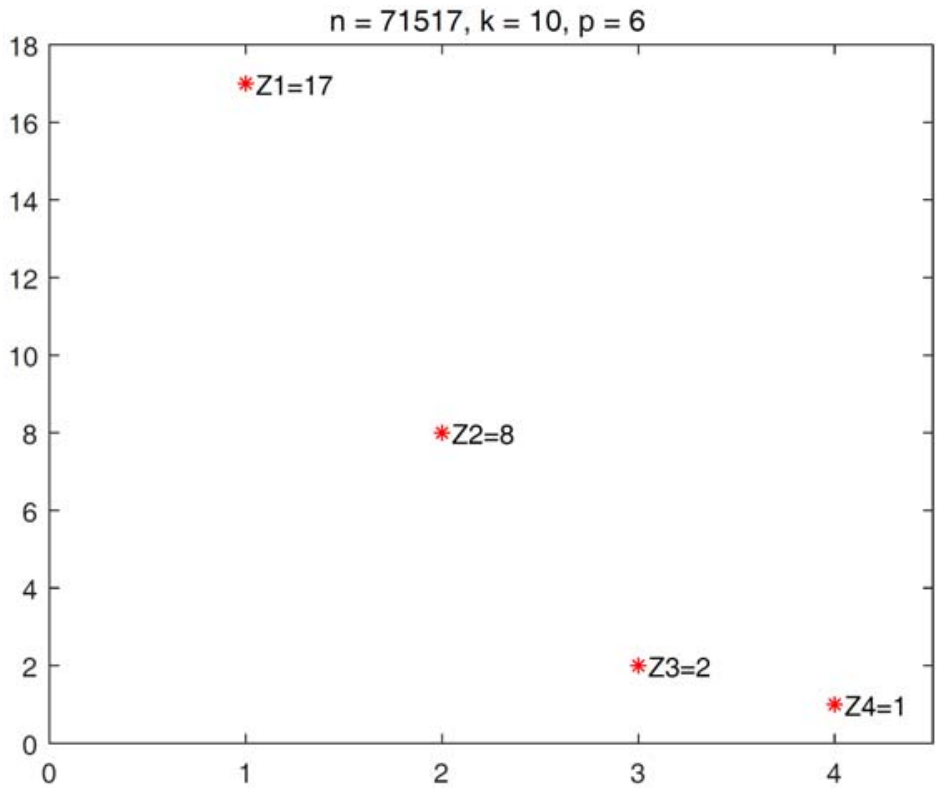

(b) $n=71517, k=10, p=6$

Figure 1. Two sets of $n, k, p$, which satisfy the assumptions (5). 
Example 3. Take $n=283, k=3$, and $p=2$, this set doesn't satisfy the requirement of Theorem 1, that is, $p \ngtr 5$.

From Figure 2, it implies that $n=283$ in 3 -adic will converge to 4 in the sense of the $\mathcal{Z}$ transformation.

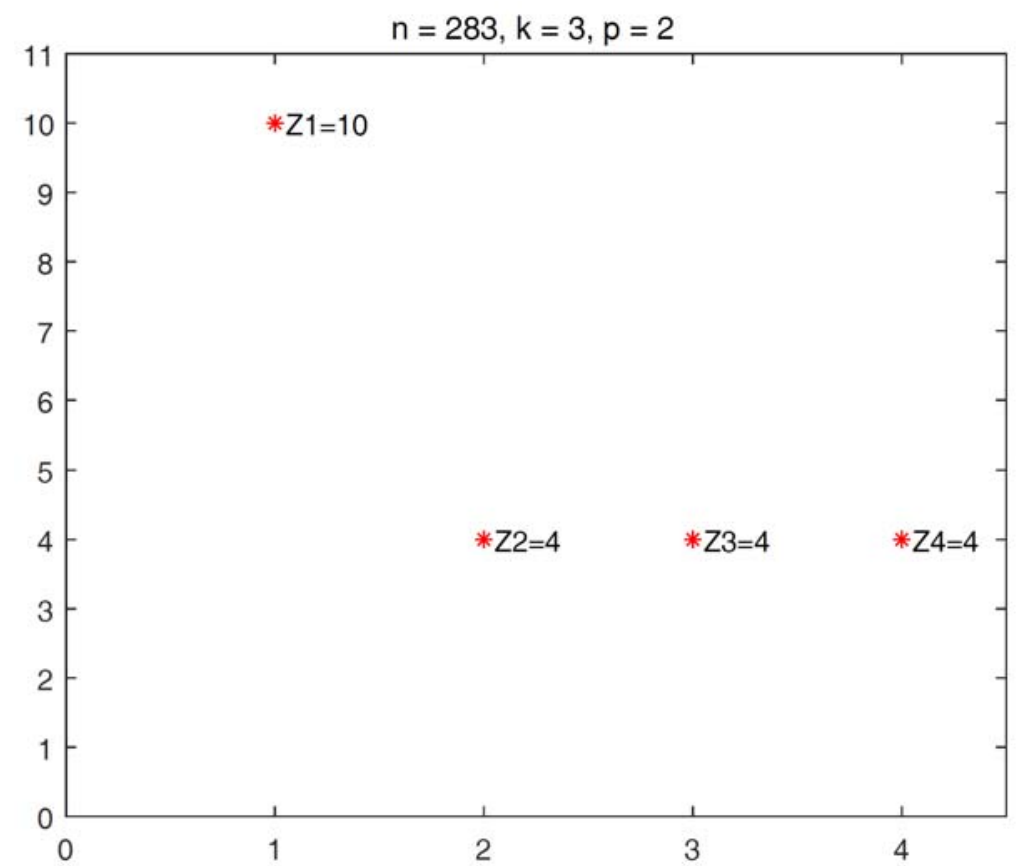

Figure 2. A set of $n, k, p$, which doesn't satisfy the assumptions.

Of course, the assumptions (5) in Theorem 1 are sufficient, but not necessary. We can see this from the following example.

Example 4. Take $n=12345, k=5$, and $p=2$. This set doesn't satisfy the requirement of Theorem 1 , but we still have that $n$ will become 1 after six times of the $\mathcal{Z}$ transformation (see Figure 3). 


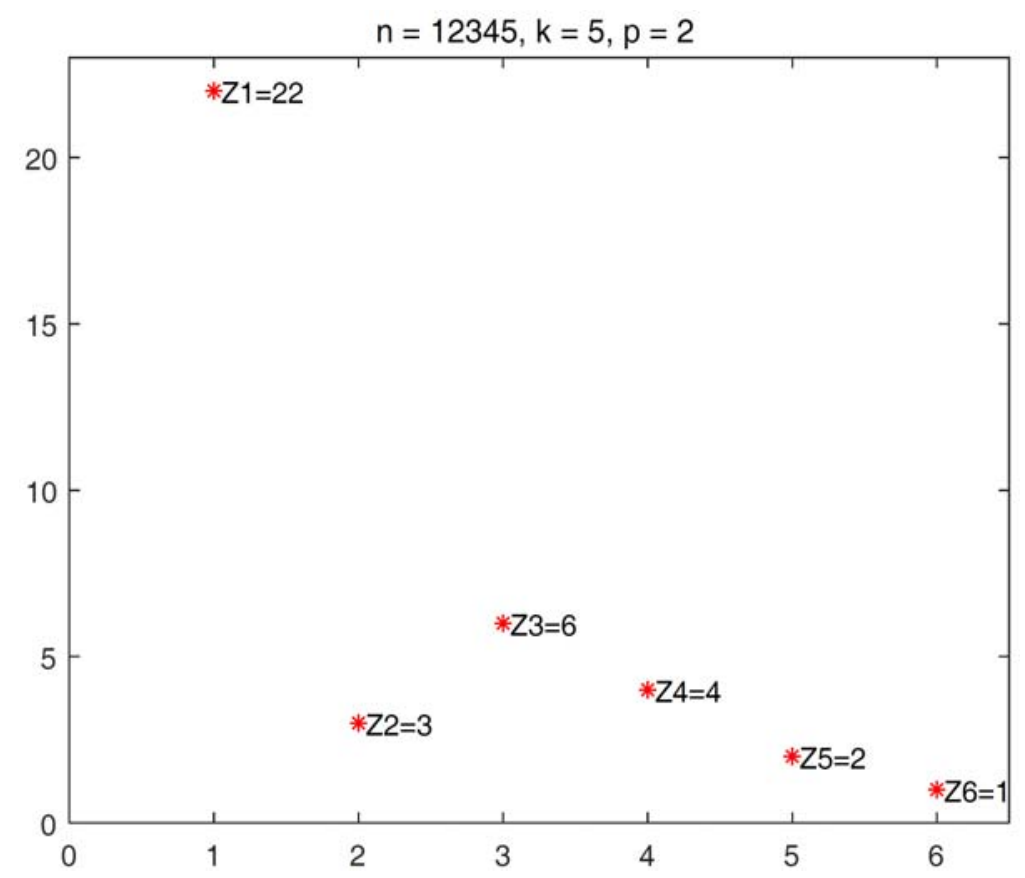

Figure 3. Another set of $n, k, p$, which doesn't satisfy the assumptions.

\section{Acknowledgement}

This article is supported by Top Disciplines (Class-A) of Zhejiang Province and Teaching Reform Project of Hangzhou Normal University.

\section{References}

[1] Cai Tianxin, The Book of Numbers, Higher Education Press, 2015, 46-47.

[2] J. C. Lagarias, The $3 x+1$ problem and its generalizations, The American Mathematical Monthly 92(1) (1985), 3-23.

DOI: https://doi.org/10.2307/2322189

[3] E. Heppner, Eine Bemerkung zum hasse-syracuse-algorithmus, Archiv der Mathematik 31(1) (1978), 317-320.

DOI: https://doi.org/10.1007/BF01226454

[4] Keith R. Matthews, The ultimate challenge: The $3 x+1$ problems, American Mathematical Society (2010), 79-103. 
[5] J. C. Lagarias, The $3 x+1$ Problem: An Annotated Bibliography, II (2000-2009), arXiv:math/0608208v6.

[6] Marc Chamberland, Averaging structure in the $3 x+1$ problem, Journal of Number Theory 148 (2015), 384-397.

DOI: https://doi.org/10.1016/j.jnt.2014.09.024

[7] Aristides V. Doumas and Vassilis G. Papanicolaou, A randomized version of the Collatz $3 x+1$ problem, Statistics \& Probability Letters 109 (2016), 39-44.

DOI: https://doi.org/10.1016/j.spl.2015.10.017

[8] Dora M. Ballesteros, Jimmy Pena and Diego Renza, A novel image encryption scheme based on Collatz conjecture, Entropy 20(12) (2018); Article 901.

DOI: https://doi.org/10.3390/e20120901 